\title{
To what extent does aortic pulse wave velocity estimate early atherosclerosis in Kurosawa and Kusanagi-hypercholesterolemic rabbits?
}

\author{
Shiho Hayashi ${ }^{1,2}$, Shinju Obara ${ }^{2}$, Masahiro Murakawa ${ }^{2}$, Akihiro Hazama ${ }^{3}$, Masahiko Kusanagi ${ }^{4}$ and \\ Shin-ichiro Katsuda ${ }^{3}$
}

Pulse wave velocity (PWV), an index of arterial stiffness, is dependent not only on structural alterations in arterial wall but also on distending pressure, which could be related to wall distensibility. We investigated the efficacy of aortic PWV (AoPWV) for detecting early to moderate atherosclerosis in Kurosawa and Kusanagi-hypercholesterolemic (KHC) rabbits. Pressure waves were recorded at the ascending, and proximal and distal abdominal aortas in 13 male normal and KHC rabbits aged 12 months under pentobarbital anesthesia when angiotensin II or sodium nitroprusside was infused intravenously. AoPWV was compared between the two rabbit groups at different mean arterial pressure (MAP) levels. Vascular diameter $\left(D_{m}\right)$ was measured using an intravascular ultrasound imaging system in the proximal thoracic and proximal abdominal aortas to determine pressure-strain elastic modulus $\left(E_{\mathrm{p}}\right)$. AoPWV was correlated to MAP in the two rabbit groups $(P<0.001)$. AoPWV was slightly but significantly greater $\left(<0.5 \mathrm{~m} \mathrm{~s}^{-1}\right)$ in the KHC rabbit group than in the normal rabbit group at MAP level $>80 \mathrm{~mm} \mathrm{Hg}(P<0.01)$. The value of $h / D_{\mathrm{m}}$ ( $h$, wall thickness) was significantly high in the KHC rabbit group at any MAP level $(P<0.001)$ in these aortic regions. There was no significant difference in $E_{\mathrm{p}}$ and $D_{\mathrm{m}}$ between the two rabbit groups at any MAP level. AoPWV showed only a slight but significant increase in the young KHC rabbits at different MAP levels, which was mainly due to the increased $h / D_{\mathrm{m}}$. AoPWV could underestimate aortic stiffness due to early to moderate atherosclerosis if AoPWV was adjusted for arterial pressure level. Hypertension Research (2011) 34, 559-564; doi:10.1038/hr.2010.273; published online 13 January 2011

Keywords: blood pressure; early to moderate atherosclerosis; Kurosawa and Kusanagi-hypercholesterolemic rabbit; pulse wave velocity; vasoactive drugs

\section{INTRODUCTION}

It is well known that pulse wave velocity (PWV) can be used as a predictor of cardiovascular mortality independently of age, blood pressure and cardiac mass in patients with hypertension, ${ }^{1-3}$ diabetes ${ }^{3,4}$ and end-stage renal failure. ${ }^{5,6} \mathrm{PWV}$ has been frequently employed in recent years in epidemiological studies, mass health screening, and diagnosis and assessment of prognosis of cardiovascular disorders, as it can be easily quantified with high reproducibility to estimate arterial stiffness.

PWV is dependent not only on pathohistological alterations in the arterial wall but also changes in arterial pressure (AP). ${ }^{7}$ Many investigators have confirmed that PWV was correlated with age, ${ }^{8-10}$ systolic blood pressure (SBP), ${ }^{11,12}$ and diastolic blood pressure (DBP). ${ }^{11,13}$ Katsuda et al. ${ }^{14}$ demonstrated previously that PWV in the entire aorta (AoPWV) in young Kurosawa and Kusanagi-hypercholesterolemic (KHC) rabbits, an animal model of familial hypercholesterolemia ${ }^{15}$ showed a slight (about $10 \%$ ) but significant increase compared with that in age-matched control rabbits, and that SBP and
DBP were also significantly higher in the KHC rabbit group than in the control group. Some investigators reported that brachial-ankle PWV (baPWV), closely associated with AoPWV measured invasively, ${ }^{16,17}$ was also useful to predict the early stage of atherosclerosis in obese young-adults ${ }^{18}$ and Type 2 diabetic patients. ${ }^{19} \mathrm{PWV}$ can be lowered in hypertensive patients after antihypertensive therapy is performed. ${ }^{20,21}$ The effects of atherosclerosis on PWV may be small at relatively early to middle stages if PWV is corrected for AP. It is necessary to clarify the efficacy of AoPWV for estimating early to moderate atherosclerosis (atheromatosis) in experimental studies before the usual clinical examinations or epidemiological investigations.

In the present study, we verified experimentally to what extent AoPWV was useful to predict atheromatosis in young KHC rabbits by comparing AoPWV between KHC and age-matched control rabbit groups before and after adjustment for AP, respectively. AoPWV was analyzed with respect to distensibility of atherosclerotic aorta at different mean arterial pressure (MAP) level altered by vasoactive drugs.

\footnotetext{
1Department of Anesthesiology, Iwaki Kyoritsu Hospital, Iwaki, Japan; ${ }^{2}$ Department of Anesthesiology, Fukushima Medical University School of Medicine, Fukushima, Japan; ${ }^{3}$ Department of Cellular and Integrative Physiology, Fukushima Medical University School of Medicine, Fukushima, Japan and 4 Japan Laboratory Animals, Inc., Tokyo, Japan Correspondence: Dr S Hayashi, Department of Anesthesiology, Iwaki Kyoritsu Hospital, 16 Kusehara Uchigo-Mimaya-cho, Iwaki, Fukushima 973-8402, Japan. E-mail: s-hayashi@iwaki-kyoritsu.iwaki.fukushima.jp

Received 20 September 2010; revised 10 November 2010; accepted 14 November 2010; published online 13 January 2011
} 


\section{METHODS}

Animals

Thirteen male KHC $(3.5 \pm 0.2 \mathrm{~kg}$ body weight, mean \pm s.d. $)$ and 13 male Japanese White rabbits $(3.8 \pm 0.4 \mathrm{~kg}$ ) (Japan Laboratory Animals, Tokyo, Japan) aged 10-12 months were used in the present study. They were given a cholesterol-free commercial rabbit chow (RC-4, Oriental Yeast, Tokyo, Japan) at $100 \mathrm{~g}$ a day and allowed freely access of tap water in an air-conditioned room (at $24 \pm 3{ }^{\circ} \mathrm{C}$ room temperature, 50-60\% relative humidity and $12 \mathrm{~L} / 12 \mathrm{D}$ light and dark cycle). The experiment was approved by the Experimental Animal Committee of Fukushima Medical University and was administered in accordance with the Guidelines for the Care and Use of Laboratory Animals published by the US National Institutes of Health.

\section{Surgical procedure}

The surgical procedure and experimental set-up was same as those reported previously. ${ }^{22}$ The rabbits were fixed in a supine position and intubated through tracheotomy under pentobarbital anesthesia at a dose of $30 \mathrm{mg} \mathrm{kg}^{-1}$ (Nembutal, Abbott Laboratories, North Chicago, IL, USA). Additional pentobarbital sodium was administered at $5 \mathrm{mg} \mathrm{kg}^{-1}$ every $15-20 \mathrm{~min}$, if necessary. Procaine chloride was applied to the incision sites to reduce pain. A catheter-tip micromanometer (3 Fr, Millar Instruments, Dallas, TX, USA) was placed in the ascending aorta. Another catheter with two micromanometers at the tip (80-mm intervals) was advanced to the abdominal aorta from the left femoral artery. One micromanometer was located at the bifurcation of the left renal artery and the other in the proximal abdominal aorta (around bifurcation of the left renal artery). Two polyethylene catheters for drug infusion were inserted from the right maxillary vein to the superior vena cava.

\section{Pressure wave recording}

Angiotensin II or sodium nitroprusside was infused in $60 \mathrm{~s}$ at doses of 50-100 $\mathrm{ng} \mathrm{kg}^{-1} \mathrm{~min}^{-1}$ and $50-100 \mu \mathrm{g} \mathrm{kg}^{-1} \mathrm{~min}^{-1}$, until the MAP level reached about 150 or $50 \mathrm{~mm} \mathrm{Hg}$, respectively. Pressure waves were simultaneously measured at the ascending aorta and proximal and distal abdominal aortas and applied into a computer through an analogue-to-digital converter (PowerLab System/16s, AD Instruments, Sydney, Australia) at intervals of $0.1 \mathrm{~ms}$ when vasoactive drugs were infused intravenously.

Measurement of vascular diameter and elastic modulus of the wall Measurement of vascular diameter and tensile characteristics in the aorta were similar to those reported previously.22 After the recording of pulse wave, intravascular ultrasound images in response to the same vasoactive drugs were recorded using an intravascular ultrasound system (In-Vision Tsunami, EndoSonic, Rancho, Cordova, CA, USA) in the origin of the descending aorta (about $20 \mathrm{~mm}$ distal to the bifurcation of the left subclavian artery) and proximal abdominal aorta (between the bifurcation of the superior mesenteric and left renal arteries) almost at the same MAP and heart rate levels as those used for determination of PWV. Systolic $\left(D_{\mathrm{s}}\right)$ and diastolic $\left(D_{\mathrm{d}}\right)$ internal diameters for five successive pulse were measured using a computer (PC-LL9307D, NEC Personal Products, Tokyo, Japan) at MAP levels of 60, 80, 100, 120 and $140 \mathrm{~mm} \mathrm{Hg}$ in the ascending aorta after the video images had been digitized through an analogue-to-digital converter (GV-MVP/RZ, I/O Data System, Kanazawa, Japan). The pressure-strain elastic modulus $\left(E_{\mathrm{P}}\right)$ was calculated as $E_{\mathrm{p}}=\mathrm{PP} /\left(\left(D_{\mathrm{s}}-D_{\mathrm{d}}\right) / D_{\mathrm{m}}\right)$, where PP and $D_{\mathrm{m}}$ denote pulse pressure and mean diameter $\left(D_{\mathrm{s}}+D_{\mathrm{d}}\right) / 2$, respectively. ${ }^{23} \mathrm{PP}$ in the ascending aorta and proximal abdominal aorta were used for calculation of $E_{\mathrm{p}}$ in the origin of the descending aorta and proximal abdominal aorta, respectively.

\section{Determination of PWV}

The distance between the micromanometer at the ascending aorta and the entry site of the catheter-transducer at the left femoral artery $\left(\Delta D_{\mathrm{AA}-\text { fem }}\right)$ was precisely measured in situ using a string after euthanizing by overdose injection of pentobarbital sodium. The distance between the two micromanometers placed at the ascending aorta and distal abdominal aortas $(\Delta D)$ was calculated as $\Delta D_{\text {AA-fem }}-80 \mathrm{~mm}$. The rising time of the pressure waves was determined as the second peak of the second derivative of the original waves. ${ }^{14}$ AoPWV was calculated as $\Delta D / \Delta T$, where $\Delta T$ was the difference in the rise time of pressure waves recorded at the ascending and distal abdominal aortas.

\section{Measurement of percent lesioned area and wall thickness}

The aorta was removed from its origin to the bifurcation of the common iliac arteries and xerox copied. The outline of the aorta and atheromatous plaques was carefully traced and fed into a personal computer through an image scanner. The lesioned and intact areas were displayed in black and white, respectively. The area of the plaques $\left(A_{\text {lesion }}\right)$ and the entire inner surface of the aorta $\left(A_{\text {total }}\right)$ were calculated using freely available software (NIH Scion Image, Frederick, MD, USA). The percent lesioned area was determined as $A_{\text {lesion }} /$ $A_{\text {total }} \times 100$. The aorta was stored at $-85^{\circ} \mathrm{C}$ and thawed rapidly at $37^{\circ} \mathrm{C}$ before measurement of wall thickness.

Circumferential strips $3 \mathrm{~mm}$ in width were excised at in the proximal thoracic and proximal abdominal aortas where intravascular ultrasound images had been recorded, and weighed precisely with a precision balance. The wall thickness $(h)$ was calculated as $h=W /\left(1.06 \pi \times D_{\mathrm{m}} \times W_{\mathrm{d}}\right)$, where $1.06,{ }^{24} W$ and $W_{\mathrm{d}}$ denoted the density of the wall $\left(\mathrm{g} \mathrm{cm}^{-3}\right)$, sample weight $(\mathrm{kg})$ and sample width $(\mathrm{mm})$, respectively.

\section{Histological sections}

After weighing the samples, wall strips were fixed in $10 \%$ neutral buffered formalin solution. The strips were embedded in paraffin and sectioned at $5 \mu \mathrm{m}$. Sections were stained with Elastica-van Gieson stain.

\section{Statistical analysis}

PWV, $E_{\mathrm{p}}, D_{\mathrm{m}}, h$ and $h / D_{\mathrm{m}}$ at different MAP levels were tested by repeated measures two-way analysis of variance. If significant differences were observed, PWV, $E_{\mathrm{p}}, D_{\mathrm{m}}, h$ and $h / D_{\mathrm{m}}$ at MAP levels of $60,80,100,120$ and $140 \mathrm{~mm} \mathrm{Hg}$ were compared between normal and KHC rabbit groups using Scheffe's multicomparison test. Correlation analysis was performed between MAP and PWV, and between MAP and $E_{\mathrm{p}}, D_{\mathrm{m}}, h$ and $h / D_{\mathrm{m}}$, respectively. Pearson's simple correlation coefficient was calculated and tested using Student's $t$-test. Significance was accepted at $P<0.05$.

\section{RESULTS}

Pulse wave velocity before infusion of vasoactive drugs

Table 1 illustrates AoPWV, SBP, DBP and MAP before the infusion of vasoactive drugs in the $\mathrm{KHC}$ and control rabbit groups. AoPWV $(P<0.001)$, SBP $(P<0.001)$, DBP $(P<0.001)$ and MAP $(P<0.001)$ were significantly greater in the KHC rabbit group than in the control rabbit group.

\section{Relationships between MAP and PWV}

Figure 1 shows the correlation of AoPWV with MAP (a), SBP (b), DBP (c) and PP (d) in the normal and KHC rabbit groups. AoPWV was significantly correlated with MAP level in the normal $(r=0.95$; $P<0.001)$ and KHC $(r=0.95 ; P<0.001)$ rabbit groups. There were also significant positive correlations between SBP and AoPWV ( $r=0.97 ; P<0.001$ and $r=0.95 ; P<0.001$, respectively for the normal and KHC rabbit groups), between DBP and AoPWV ( $r=0.96$;

Table 1 AoPWV, SBP, DBP, MAP and PP in normal and KHC rabbit groups before the infusion of vasoactive drugs

\begin{tabular}{llllll}
\hline & \multicolumn{1}{c}{ AoPWV } & \multicolumn{1}{c}{$S B P$} & \multicolumn{1}{c}{ DBP } & \multicolumn{1}{c}{$M A P$} & \multicolumn{1}{c}{$P P$} \\
\hline Normal & $5.30 \pm 0.24$ & $126.7 \pm 5.6$ & $103.4 \pm 5.2$ & $114.6 \pm 5.7$ & $23.3 \pm 3.9$ \\
KHC & $6.29 \pm 0.41^{* * *}$ & $141.2 \pm 6.1^{* * *}$ & $113.8 \pm 4.1^{* * *}$ & $128.1 \pm 4.7^{* * *}$ & $27.4 \pm 4.0^{* *}$ \\
\hline
\end{tabular}

Abbreviations: AoPWV, aortic pulse wave velocity; DBP, diastolic blood pressure; $\mathrm{KHC}$, Kurosawa and Kusanagi-hypercholesterolemic; MAP, mean arterial pressure; PP, pulse pressure; SBP, systolic blood pressure.

The data are mean \pm s.d.

The data are mear

${ }^{* *} P<0.01$. 

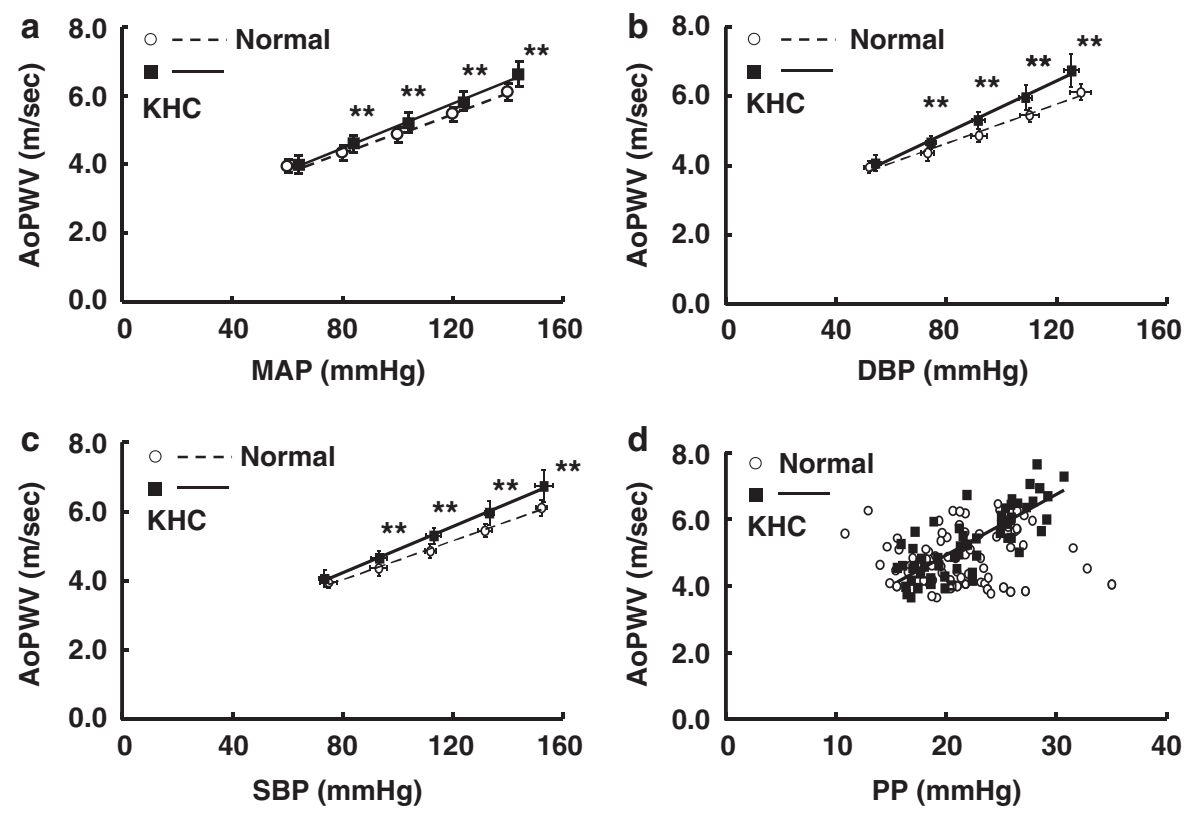

Figure 1 Correlation diagrams between MAP and AoPWV (a), between SBP and AoPWV (b), between DBP and AoPWV (c) and between PP and AoPWV (d) in normal and KHC rabbit groups. The data are mean \pm s.d. SBP and DBP are measured at MAP levels at 60, 80, 100,120 and $140 \mathrm{~mm} \mathrm{Hg}$ and averaged within each MAP level, respectively. PP is determined at these MAP levels. Correlation coefficient, significant difference and regression formula are as follows: (a) $r=0.95, P<0.001, y=0.027 x+2.21$, for normal rabbit group. $r=0.95, P<0.001, y=0.033 x+1.86$, for KHC rabbit group. (b) $r=0.97$, $P<0.001, y=0.028 x+1.78$, for normal rabbit group. $r=0.95, P<0.001, y=0.034 x+1.56$, for KHC rabbit group. (c) $r=0.96 ; P<0.001, y=0.028 x+2.36$, for normal rabbit group. $r=0.94 ; P<0.001, y=0.038 x+1.93$, for $\mathrm{KHC}$ rabbit group. (d) $r=0.11 ; P>0.05$, for normal rabbit group. $r=0.79 ; P<0.001$, $y=0.183 x+1.29$, for the KHC rabbit group. MAP, mean arterial pressure; SBP, systolic blood pressure; DBP, diastolic blood pressure; PP, pulse pressure; AoPWV, aortic pulse wave velocity.

$P<0.001$ and $r=0.94 ; P<0.001$, respectively for the normal and KHC rabbit groups), and between PP and AoPWV ( $r=0.79 ; P<0.001$ for the KHC rabbit group), whereas AoPWV showed no correlation with $\mathrm{PP}$ in the normal rabbit group $(r=0.11 ; P>0.05)$. AoPWV in the KHC rabbit group showed only a slight but significant increase at MAP levels of $80 \mathrm{~mm} \mathrm{Hg} \quad(P<0.01), \quad 100 \mathrm{~mm} \mathrm{Hg} \quad(P<0.01)$, $120 \mathrm{~mm} \mathrm{Hg}(P<0.01)$ and $140 \mathrm{~mm} \mathrm{Hg}(P<0.01)$ when compared with that in the normal rabbit group. The difference in AoPWV between the two rabbit groups tended to increase gradually as the MAP level increased.

Relationships between MAP and $E_{\mathrm{p}}, h, D_{\mathrm{m}}, h / D_{\mathrm{m}}$

Figure 2 shows the correlation between MAP and $E_{\mathrm{p}}(\mathbf{a})$, between MAP and $h(\mathbf{b})$, between MAP and $D_{\mathrm{m}}(\mathbf{c})$ and between MAP and $h / D_{\mathrm{m}}(\mathbf{d})$ in the proximal thoracic aorta. $E_{\mathrm{p}}$ was significantly correlated with MAP $(r=0.58 ; P<0.001$ and $r=0.81 ; P<0.01$, respectively for normal and KHC rabbit groups). $D_{\mathrm{m}}$ was also significantly correlated with MAP ( $r=0.73 ; P<0.001$ and $r=0.59 ; P<0.001$, respectively for normal and KHC rabbit groups). However, there were no significant differences in $E_{\mathrm{p}}$ and $D_{\mathrm{m}}$ between the two rabbit groups at any MAP level in this aortic region. There were significant negative correlation between MAP and $h(r=-0.74 ; P<0.001$ and $r=-0.30 ; P<0.001$, respectively for normal and KHC rabbit groups), between MAP and $h / D_{\mathrm{m}}(r=-0.81 ; P<0.001$ and $r=-0.52 ; P<0.001$, respectively for normal and KHC rabbit groups). The value of $h$ and $h / D_{\mathrm{m}}$ were significantly greater in the KHC rabbits group at any MAP level $(P<0.001)$.

Figure 3 depicts the correlation between MAP with $E_{\mathrm{p}}(\mathbf{a})$, between MAP and $h(\mathbf{b})$, between MAP and $D_{\mathrm{m}}(\mathbf{c})$ and between MAP and $h / D_{\mathrm{m}}(\mathbf{d})$ in the proximal abdominal aorta. The value of $E_{\mathrm{p}}(r=0.74$; $P<0.001$ and $r=0.82 ; P<0.001$, respectively for normal and $\mathrm{KHC}$ rabbit groups $)$ and $D_{\mathrm{m}}(r=0.69 ; P<0.001$ and $r=0.61 ; P<0.001$, respectively for normal and KHC rabbit groups) showed significant positive correlation with MAP. No significant difference in $E_{\mathrm{p}}$ and $D_{\mathrm{m}}$ was observed between the two rabbit groups at any MAP level. There was a significant negative correlation between $h$ and MAP $(r=-0.55$; $P<0.001$ and $r=-0.27 ; P<0.05$, respectively for normal and KHC rabbit groups). The value of $h / D_{\mathrm{m}}$ also showed significant negative correlation with MAP $(r=-0.72 ; P<0.001$ and $r=-0.49 ; P<0.001$, respectively for normal and KHC rabbit groups). The values of $h$ and $h / D_{\mathrm{m}}$ were significantly greater in the KHC rabbits group than in the normal rabbit group at any MAP levels $(P<0.01$ and $P<0.001$, respectively for $h$ and $\left.h / D_{\mathrm{m}}\right)$.

\section{Macroscopic and microscopic findings of the aorta}

Figure 4 shows examples of photocopies of the aorta in KHC rabbits aged 12 months. In the KHC rabbits atheromatous plaques were located in the ascending aorta and around bifurcations of the main branch arteries, such as the intrecostal, celiac, mesenteric and renal arteries. The extent of sclerotic lesions was considerably varied. Percent lesioned area was $54.3 \pm 20.5 \%$ (mean \pm s.d.). Photomicrographs of transverse histological sections in the proximal thoracic (a) and proximal abdominal (b) aortas are shown in Figure 5. Marked intimal thickening with abundant foam cells were dominant in the tested regions. Fibrous proliferation was partly observed among foam cells. The inner elastic lamina was partly injured and duplicated.

\section{DISCUSSION}

PWV has been frequently employed as a direct measure of arterial stiffness in recent years for mass physical screening, diagnosis and prognosis of cardiovascular disease. Arterial stiffness is an independent 

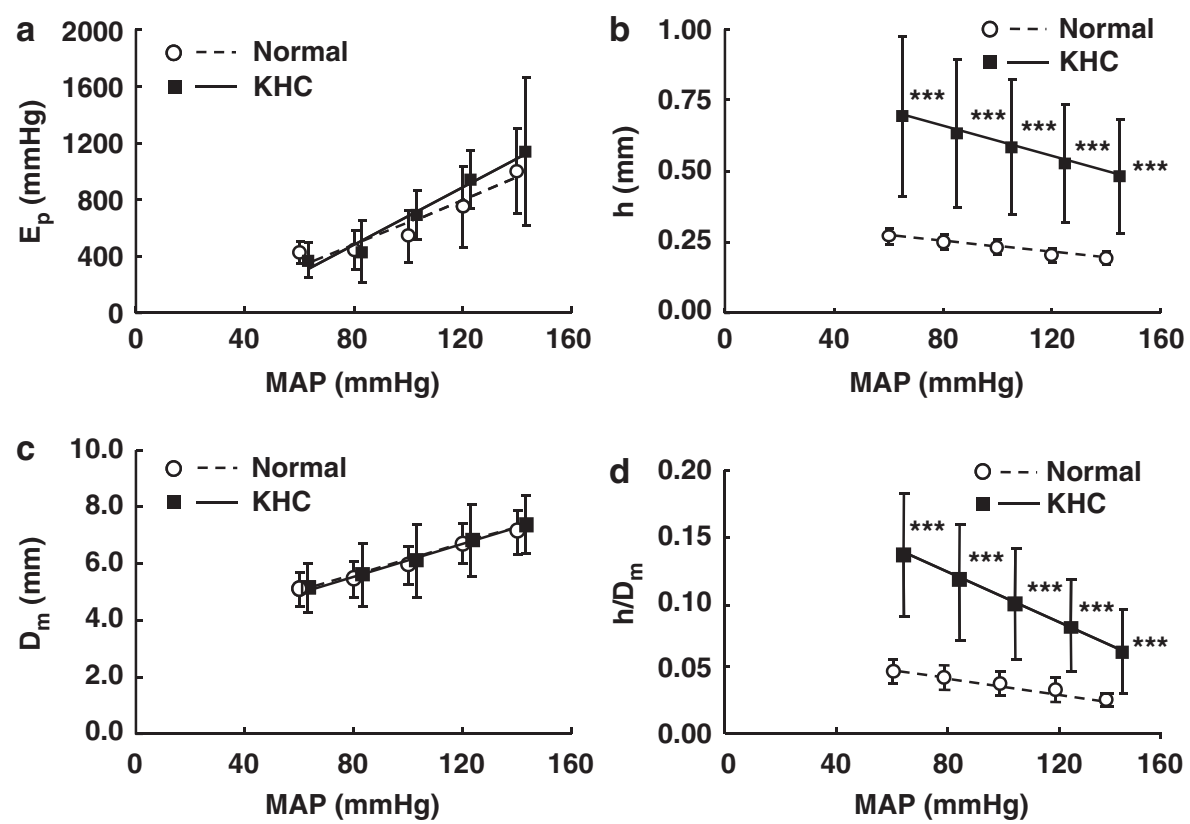

Figure 2 Correlation diagrams between MAP and $E_{\mathrm{p}}(\mathbf{a})$, between MAP and $h(\mathbf{b})$, between MAP and $D_{\mathrm{m}}(\mathbf{c})$ and between MAP and $h / D_{\mathrm{m}}(\mathbf{d})$ in the proximal thoracic aorta in the normal and $\mathrm{KHC}$ rabbit groups. The data are mean \pm s.d. Correlation coefficient, significant difference and regression formula are as follows: (a) $r=0.58, P<0.001, y=7.22 x-91.72$, for normal rabbit group. $r=0.81, P<0.01, y=10.21 x-337.94$, for KHC rabbit group. (b) $r=-0.74$, $P<0.001, y=-0.001 x+0.38$, for normal rabbit group. $r=-0.30, P<0.001, \quad y=-0.003 x+0.86$, for KHC rabbit group. (c) $r=0.73, P<0.001$, $y=0.025 x+3.32$, for normal rabbit group. $r=0.59, P<0.001, y=0.029 x+3.27$, in KHC rabbit group. (d) $r=-0.81, P<0.001, y=-0.0003 x+0.072$, in normal rabbit group. $r=-0.52, P<0.001, y=-0.0009 x+0.19$, in $\mathrm{KHC}$ rabbit group. $E_{\mathrm{p}}$, pressure-strain elastic modulus; $D_{\mathrm{m}}$, mean diameter; $h$, the thickness of the wall; MAP, mean arterial pressure.
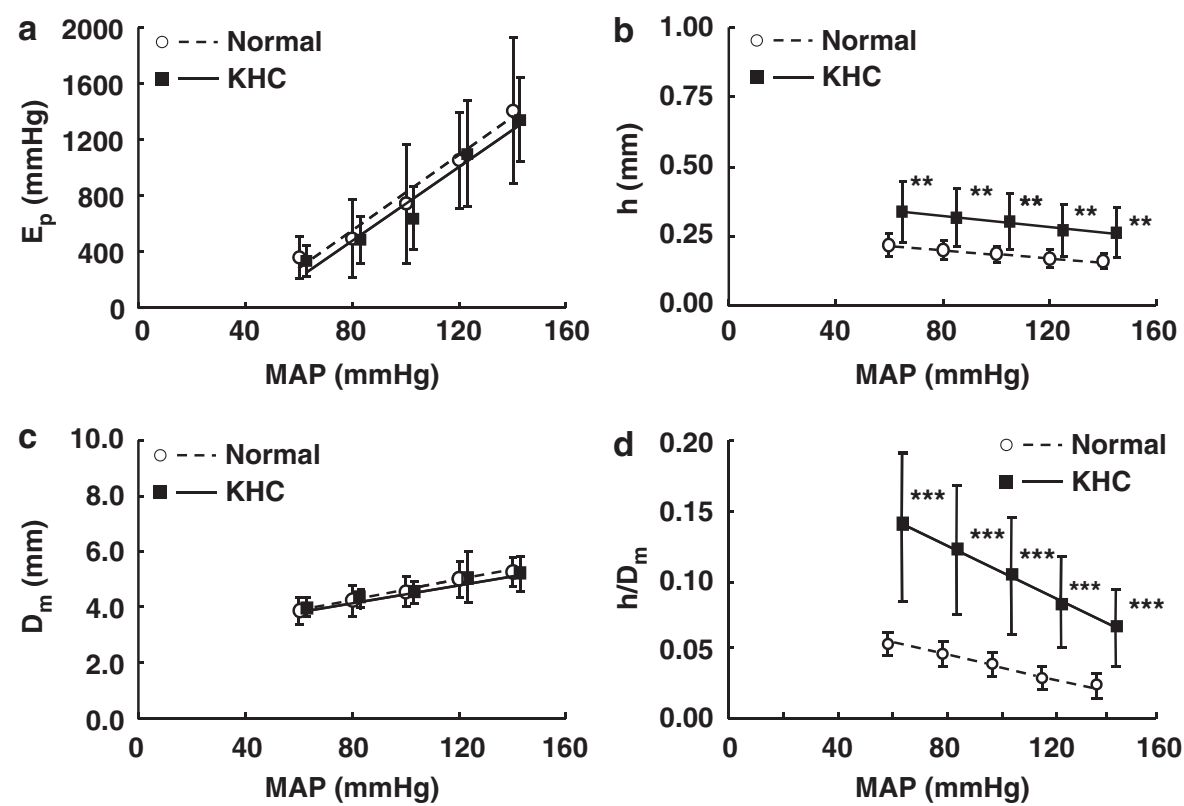

Figure 3 Correlation diagrams between MAP and $E_{\mathrm{p}}(\mathbf{a})$, between MAP and $h(\mathbf{b})$, between MAP and $D_{\mathrm{m}}(\mathbf{c})$ and between MAP and $h / D_{\mathrm{m}}(\mathbf{d})$ in the proximal abdominal aorta in the normal and KHC rabbit groups. The data are mean \pm s.d. Correlation coefficient, significant difference and regression formula are as follows: (a) $r=0.74, P<0.001, y=13.31 x-519.82$, for normal rabbit group. $r=0.82, P<0.001, y=13.14 x-572.41$, for KHC rabbit group. (b) $r=-0.55$, $P<0.001, y=-0.0007 x+0.29$, for normal rabbit group. $r=-0.27, P<0.05, y=-0.001 x+0.40$, for $\mathrm{KHC}$ rabbit group. (c) $r=0.69, P<0.001$, $y=0.019 x+2.75$, for normal rabbit group. $r=0.61, P<0.001, y=0.012 x+3.01$, in KHC rabbit group. (d) $r=-0.72, P<0.001, y=-0.0003 x+0.065$, in normal rabbit group. $r=-0.49, P<0.001 y=-0.0004 x+0.11$, in $\mathrm{KHC}$ rabbit group. $E_{\mathrm{p}}$, pressure-strain elastic modulus; $D_{\mathrm{m}}$, mean diameter; $h$, the thickness of the wall; MAP, mean arterial pressure.

predictor of cardiovascular ${ }^{25}$ and all-cause ${ }^{26}$ mortality, which is associated with age, ${ }^{8-10}$ atherosclerosis, ${ }^{27}$ hypertension, ${ }^{1,2}$ Type 2 diabetes $^{4}$ and end-stage renal failure. ${ }^{5,6}$
PWV can be theoretically expressed by the Moens-Korteweg equation; ${ }^{28,29} \mathrm{PWV}=k \sqrt{ } E h / \rho D$, where $k, E, h, \rho$ and $D$ are a constant, elastic modulus, wall thickness, the density of the blood and arterial 
internal diameter, respectively. PWV is dependent not only on structural alterations of the arterial wall such as atherosclerosis but also on distending pressure, which could be directly related to the internal diameter of the artery or the elastic modulus of the artery wall.

In the present study, AoPWV in the KHC rabbit group showed only a slight but significant increase at MAP levels $>80 \mathrm{~mm} \mathrm{Hg}$ (at most $<0.5 \mathrm{~m} \mathrm{~s}^{-1}$ at each MAP level) when compared with that in the agematched control rabbit group at the same MAP level, even though $54 \%$ of the aortic intimal surface was covered with atheromatous plaques on average. In contrast, AoPWV was significantly greater by about $1.0 \mathrm{~m} \mathrm{~s}^{-1}$ in the KHC rabbit group than in the control rabbit group before the infusion of vasoactive drugs, indicating that the significantly increased AP level in the KHC rabbit group contributed to elevate AoPWV. Similar findings have been reported previously in KHC rabbits. ${ }^{14}$ Sclerotic lesions in young-adult KHC rabbits consisted mainly of abundant foam cells, which were thought to endow the wall with viscoelastic properties. Katsuda et al..$^{30}$ demonstrated previously that the aortic walls of KHC rabbits aged 10-12 months were less rigid in an ex vivo tensile study. Newman et al. ${ }^{31}$ also observed similar findings in cholesterol-fed cockerels. Though the value of $\rho$ was not measured in the present study, Hasegawa et al. ${ }^{32}$ reported no significant difference in $\rho$ between normal and heritable hyperlipidemic rabbit groups. We consider that the significantly increased $h$ at any MAP level likely contributes to the slight but significant increase in AoPWV in the KHC rabbit group, because there were no significant

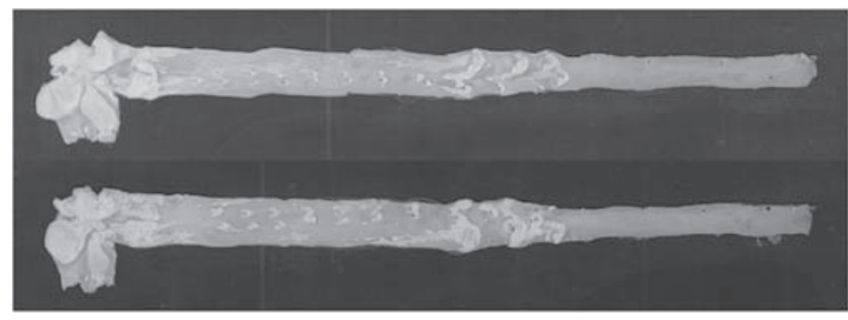

$20 \mathrm{~mm}$

Figure 4 Example of photocopies of the aorta in KHC rabbits aged 12 months. differences in $E_{\mathrm{p}}$ and $D_{\mathrm{m}}$ at any MAP level between the two rabbit groups. Appearance of significant statistical differences in AoPWV between the two rabbit groups would be partly due to the considerably smaller variation in AoPWV at any MAP level, though the difference in AoPWV between the two rabbit groups was very slight. It is also considered that effect of AP on the aortic wall is buffered by cholesterol-rich atheromatous plaques, which would be partly responsible for minimizing difference in AoPWV between the two rabbit groups at the same MAP level.

Early detection of cardiovascular risk factors, such as hyperlipidemia, obesity and high blood pressure were identified in childhood ch-35 $^{33}$ or adolescence ${ }^{18,36}$ and establishment of appropriate primary prevention programs could influence health in later life. There are pathological studies indicating that atherosclerotic lesions begin even in childhood. ${ }^{37,38}$ Several investigators have demonstrated that baPWV showed a significant correlation with MAP in young adults $^{18,36}$ and with SBP and DBP in children. ${ }^{35,39}$ In the Bogalusa Heart Study, Li et al. ${ }^{34}$ reported that childhood blood pressure was a predictor of arterial stiffness in adulthood. AP level is thought to contribute considerably to arterial stiffness. To what extent PWV is useful to assess relatively early atherosclerotic lesion per $s e$ ?

Carotid intima-media thickness has frequently been measured using ultrasonography to estimate early atherosclerosis. Strong positive correlation of intima-media thickness with carotid-femoral PWV has been observed in large population cohort studies ${ }^{27}$ and in patients with diabetes. ${ }^{19,40}$ baPWV $^{16,17}$ was also useful to predict the early stages of atherosclerosis. ${ }^{18,19}$ In contrast, Nishi et al. ${ }^{41}$ also observed a significant but poor correlation between baPWV and intima-media thickness in subjects with diabetes and no significant relationship with baPWV in the presence of plaques. Further investigation is needed to assess usefulness of baPWV for detection of early atherosclerosis.

Hypertension has been well shown to be one of the major risk factors for cardiovascular events. Therefore, we also raise the question of whether PWV should be corrected for AP or not? Hasegawa ${ }^{42}$ previously established a precise nomogram of carotid-femoral PWV adjusted for DBP, age and sex in large number of healthy subjects. They used a PWV corrected for DBP at $80 \mathrm{mmHg}$ using their nomogram for diagnosis and clinical follow-up of vascular disease,
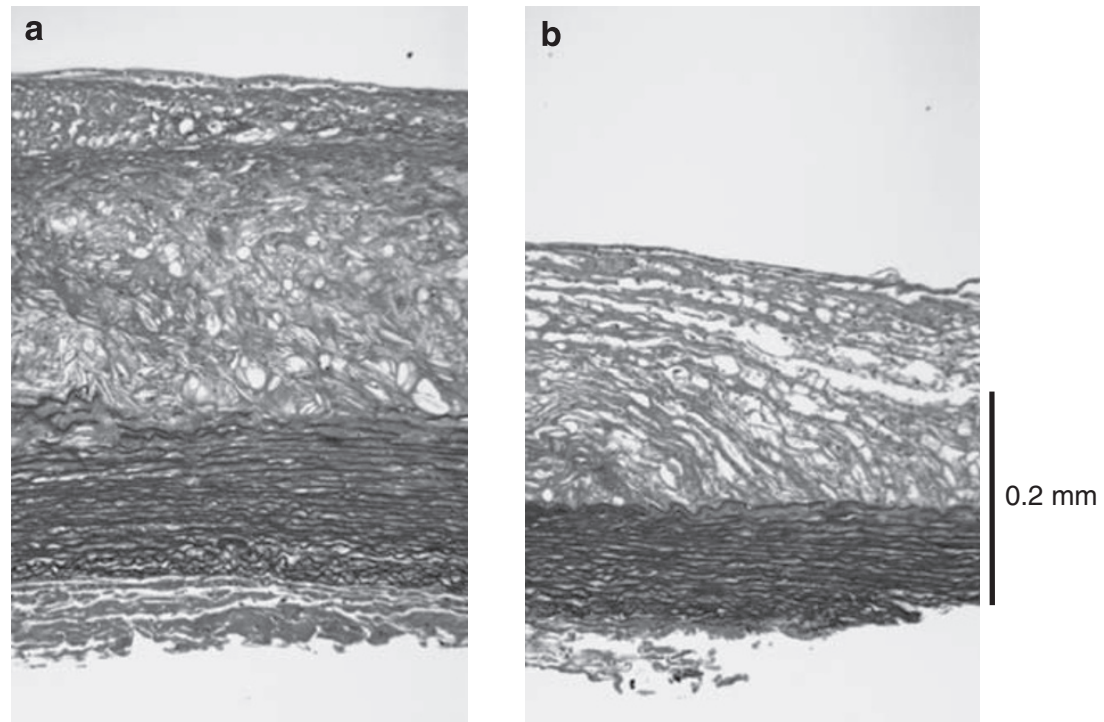

Figure 5 Photomicrographs of transverse histological sections in the proximal thoracic (a) and proximal abdominal (b) aortas in KHC rabbits aged 12 months. 
which made it possible to estimate arterial stiffness without the influence of distending pressure and to compare cardiovascular findings among subjects. The corrected PWV, however, would not be effective for assessing the rigidity of vessel walls due to atherosclerosis under conditions of hypertension. Zureik et al. ${ }^{43}$ reported that the significant relationship between carotid intima-media thickness and carotid-femoral PWV disappeared after further adjustment for age and blood pressure, while the relationship remained significant after adjusting for gender. In recent years, PWV has been little adjusted in most countries, although a nomogram of baPWV ${ }^{44}$ corrected for AP, age and gender has been established. Antihypertensive therapy could lower PWV. ${ }^{20,21}$ It can be considered that adjustment of PWV for AP is not necessarily required to assess early atherosclerosis in the sight of predicting cardiovascular risk.

In conclusion, AoPWV could underestimate alteration in aortic stiffness due to atherosclerotic plaques at the early to middle stages if AoPWV was corrected for AP. It is desirable to employ AoPWVs with and without adjusting for AP to estimate arterial stiffness precisely. We believe that the present study will offer beneficial information for assessment of early to moderate atherosclerosis using PWV for diagnosis and clinical follow-up in patients treated for hypertension.

\section{CONFLICT OF INTEREST}

The authors declare no conflict of interest.

\section{ACKNOWLEDGEMENTS}

We are grateful to Professor Hitoshi Yokoyama, Dr Hirono Satokawa and Dr Shinya Takase, Department of Cardiovascular Surgery, Fukushima Medical University School of Medicine, Japan for kindly offering IVUS system.

1 Blacher J, Asmar R, Djane S, London GM, Safar ME. Aortic pulse wave velocity as a marker of cardiovascular risk in hypertensive patients. Hypertension 1999; 33: 1111-1117.

2 Boutouyrie P, Tropeano AI, Asmar R, Gautier I, Benetos A, Locally P, Laurent S. Aortic stiffness is an independent predictor of primary coronary events in hypertensive patients. A longitudinal study. Hypertension 2002; 39: 10-15.

3 Liu XN, Gao HQ, Li BY, Cheng M, Ma YB, Zhang ZM, Gao XM, Liu YP, Wang M. Pulse wave velocity as a marker of arteriosclerosis and its comorbidities in Chinese patients. Hypertens Res 2007; 30: 237-242.

4 Cruickshank K, Riste L, Anderson SG, Wright JS, Dunn G, Gosling RG. Aortic pulsewave velocity and its relationship to mortality in diabetes and glucose intolerance. An integrated index of vascular function? Circulation 2002; 106: 2085-2090.

5 Blacher J, Guerin AP, Pannier B, Marchais SJ, Safar ME, London GM. Impact of aortic stiffness on survival in end-stage renal disease. Circulation 1999; 99: 2434-2439.

6 Blacher J, Safar ME, Guerin AP, Pannier B, Marchais SJ, Sylvian J, London GM. Aortic pulse wave velocity index and mortality in end-stage renal disease. Kidney Int 2003; 63: $1852-1860$.

7 Cohn JN. Arterial stiffness, vascular disease, and risk of cardiovascular events. Circulation 2006; 113: 601-603.

8 Tomiyama H, Yamashina A, Arai T, Hirose K, Koji Y, Chikamori T, Hori S, Yamamoto Y, Doba N, Hinohara S. Influences of age and gender on results of noninvasive brachialankle pulse wave velocity measurement-a survey of 12517 subjects. Atherosclerosis 2003; 166: 303-309.

9 McEniery CM, Yasmin, Hall IR, Qasem A, Wilkinson IB, Cockcroft JR. Normal vascular aging: differential effects on wave reflection and aortic pulse wave velocity. The AngloCardiff Collaborative Trial (ACCT). J Am Coll Cardiol 2005; 46: 1753-1760.

10 O'Rourke MF, Hashimoto J. Mechanical factors in arterial aging. A clinical perspective. J Am Coll Cardiol 2007; 50: 1-13.

11 Yasmin, Brown MJ. Similarities and differences between augmentation index and pulse wave velocity in the assessment of arterial stiffness. QJ Med 1999; 92: 595-600.

12 Kim EJ, Park CG, Park JS, Suh SY, Choi CU, Kim JW, Kim SH, Lim H, Rha SW, Seo HS, Oh DJ. Relationship between blood pressure parameters and pulse wave velocity in normotensive and hypertensive subjects: invasive study. J Hum Hypertens 2007; 21: 141-148.

13 Nürnberger J, Dammer S, Saez AO, Philipp T, Schäfers RF. Diastolic blood pressure is an important determinant of augmentation and pulse wave velocity in young, healthy males. J Hum Hypertens 2003; 17: 153-158.

14 Katsuda S, Miyashita H, Hasegawa M, Machida N, Kusanagi M, Yamasaki M, Hazama A. Characteristic change in local pulse wave velocity in different segments of the atherosclerotic aorta in KHC rabbits. Am J Hypertens 2004; 17: 181-187.
15 Kurosawa T, Kusanagi M, Yamasaki Y, Senga Y, Yamamoto T. A new mutant rabbit with hypercholesterolemia and atherosclerotic lesions produced by serial breeding. Lab Anim Sci 1995; 45: 385-392.

16 Yambe M, Tomiyama H, Hirayama Y, Gulniza Z, Takata Y, Koji Y, Motobe K, Yamashina A. Arterial stiffening as a possible risk factor for both atherosclerosis and diastolic heart failure. Hypertens Res 2004; 27: 625-631.

17 Yamashina A, Tomiyama H, Takeda K, Tsuda H, Arai T, Hirose K, Koji Y, Hori S, Yamamoto $Y$. Validity, reproducibility, and clinical significance of noninvasive brachialankle pulse wave velocity measurement. Hypertens Res 2002; 25: 359-364.

18 Shiotani A, Motoyama M, Matsuda T, Miyanishi T. Brachial-ankle pulse wave velocity in Japanese university students. Intern Med 2005; 44: 696-701.

19 Yokoyama H, Aoki T, Imahori M, Kuramitsu M. Subclinical atherosclerosis is increased in type 2 diabetic patients with microalbuminuria evaluated by intima-media thickness and pulse wave velocity. Kidney Int 2004; 66: 448-454.

20 Uchida H, Nakamura Y, Kaihara M, Sugimoto T, Norii H, Sasaki M, Sato H, Makino H. Practical efficacy of telmisartan for decreasing morning home blood pressure and pulse wave velocity in patients with mild-to-moderate hypertension. Hypertens Res 2004; 27: 545-550.

21 Morimoto S, Yano Y, Maki K, Sawada K. Renal and vascular protective effects of telmisartan in patients with essential hypertension. Hypertens Res 2006; 29: 567-572.

22 Obara S, Hayashi S, Hazama A, Murakawa M, Katsuda S. Correlation between augmentation index and pulse wave velocity in rabbits. J Hypertension 2009; 27: 332-340.

23 Peterson LH, Jensen RE, Parnell J. Mechanical properties of arteries in vivo. Circ Res 1960; 8: 622-639.

24 McDonald DA. Blood Flow in Arteries, 1st edn. Edward Arnold: London, pp 161.

25 Amar J, Ruidavets JB, Chamontin B, Drouet L, Ferrieres J. Arterial stiffness and cardiovascular risk factors in a population-based study. J Hypertens 2001; 19: 381-387.

26 Laurent S, Boutouyrie P, Asmar R, Gautier I, Laloux B, Guize L, Ducimetiere P, Benetos A. Aortic stiffness is an independent predictor of all-cause and cardiovascular mortality in hypertensive patients. Hypertension 2001; 37: 1236-1241.

27 von Popele NM, Grobbee DE, Bots ML, Asmar R, Topouchian J, Reneman RS, Hoeks APG, van der Kuip DAM, Hofman A, Witteman JCM. Association between arterial stiffness and atherosclerosis. The Rotterdam Study. Stroke 2001; 32: 454-460.

28 Möens Al. Die Pulskurve. E.J. Brill: Leiden, 1878; pp 90.

29 Korteweg DJ. ber die Fortpflanzungsgeschwindigkeit des Schalles in elastischen Rohren. Ann Phys Chem Neue Folge 1878; 5: 525-542.

30 Katsuda S, Machida N, Hasegawa M, Miyashita H, Kusanagi M, Tsubone H, Hazama A. Change in the static rheological properties of the aorta in Kurosawa and Kusanagihypercholesterolemic $(\mathrm{KHC})$ rabbits with progress of atherosclerosis. Physiol Meas 2004; 25: 505-522

31 Newman DL, Gosling RG, Bowden NLR. Changes in aortic distensibility and area ratio with the development of atherosclerosis. Atherosclerosis 1971; 14: 231-240.

32 Hasegawa M, Dobashi T, Takei S, Sakanishi A, Watanabe Y, Ito T, Shiomi M. Ultrasonic velocity measurement of the compressibility of plasma and red blood cells in normal and hyperlipidemic (WHHL) rabbits. Rep Prog Polym Phys Jpn 1988; 31: 733-736.

33 Raitakari OT, Juonala M, Kähönen M, Taittonen L, Laitinen T, Mäki-Torkko N, Jarvi-salo MJ, Uhari M, Jokinen E, Ronnemaa T, Akerblom HK, Viikari JS. Cardiovascular risk factors in childhood and carotid artery intima-media thickness in adulthood. The Cardiovascular Risk in Young Finns Study. JAMA 2003; 290: 2277-2283.

34 Li S, Chen W, Srinivasan SR, Berenson GS. Childhood blood pressure as a predictor of arterial stiffness in young adults. The Bogalusa Heart Study. Hypertension 2004; 43: 541-546.

35 Juonala M, Järvisalo MJ, Mäki-Torkko N, Kähönen M, Viikari JSA, Olli TR. Risk factors identified in childhood and decreased carotid artery elasticity in adulthood. The cardiovascular risk in Young Finns Study. Circulation 2005; 112: 1486-1493.

36 Im JA, Lee JW, Shim JY, Lee HR, Lee DC. Association between brachial-ankle pulse wave velocity and cardiovascular risk factors in healthy adolescents. J Pediatrics 2007; 150: 247-251

37 Stary HC. Lipid and macrophage accumulation in arteries of children and the development of atherosclerosis. Am J Clin Nutr 2000; 72 (Suppl): 1297S-1306S.

38 McGill Jr HC, McMahan CA, Zieske AW, Sloop GD, Walcott JV, Troxclair DA, Malcom GT, Tracy RE, Oalmann MC, Strong JP. Associations of coronary heart disease risk factors with the intermediate lesion of atherosclerosis in youth. Arterioscler Thromb Vasc Biol 2000; 20: 1998-2004.

39 Niboshi A, Hamaoka K, Sakata K, Inoue F. Characteristics of brachial-ankle pulse wave velocity in Japanese children. Eur J Pediat 2006; 165: 625-629.

40 Taniwaki H, Kawagishi T, Emoto M, Shoji T, Kanda H, Maekawa K, Nishizawa Y, Morii $\mathrm{H}$. Correlation between the intima-media thickness of the carotid artery and aortic pulse-wave velocity in patients with type 2 diabetes. Vessel wall properties in type 2 diabetes. Diabetes Care 1999; 22: 851-1857.

41 Nishi Y, Koshiyama H, Honjo S, Seino Y. Poor correlation of pulse-wave velocity and intima-media thickness in diabetic subjects. Diabetes Care 2004; 27: 2084.

42 Hasegawa M. A fundamental study on human aortic pulse wave velocity. J Jikei Med College 1970; 85: 742-760.

43 Zureik M, Temmar M, Adamopoulos C, Bureau JM, Courbon D, Thomas F, Bean K, Touboul PJ, Ducimetière $P$, Benetos $A$. Carotid plaques, but not common carotid intima-media thickness, are independently associated with aortic stiffness. $J$ Hypertens 2002; 20: 85-93.

44 Yamashina A, Tomiyama H, Arai T, Koji Y, Yambe M, Motobe H, Glunizia Z, Yamamoto Y, Hori S. Nomogram of the relation of brachial-ankle pulse wave velocity with blood pressure. Hypertens Res 2003; 26: 801-806. 